\title{
All you can tweet
}

\author{
Nature Chemistry signed up for a Twitter account in March 2009. More than 5,000 tweets later, what \\ have we learned and how do we use it?
}

When cramming an informative and selfcontained message into only 140 characters (including spaces!), clarity is a virtue.

With subjects that sometimes rely on specialist language, such as chemistry, composing tweets can be quite challenging.

We use Twitter to point out any interesting chemistry-related content, including papers, news stories and blog posts.

We retweet chemistry-related job opportunities and internships that we think might be of interest to our followers.

Our tweets range from serious topics (chemical safety) to the light-hearted (such as chemistry-themed music parodies: http://bit.ly/cmmbk).

We share our Articles and other content from Nature Chemistry on Twitter, but that's only a small fraction of what we tweet about.

Journal Twitter streams - especially automated ones - full of just their own content can resemble RSS feeds and are a missed opportunity.

There are quite a few chemistry journals with an active presence on Twitter and we maintain a list of them (http://bit.ly/chemjnls).

A handful of chemistry editors and journalists are on Twitter and following them gives you a behind-the-scenes look at the publishing world.

Twitter provides a direct - and effectively instant - two-way connection between the journal's editors and its followers.

All of the Nature Chemistry editors (past and present) have contributed to the journal's Twitter feed, albeit some more than others.

Ideas for research highlights come from many different sources, including tweets by chemists about papers that catch their eye.

Twitter can be a great way to crowd-source answers to a question - there are lots of experts (and others) just waiting to chime in.

Have a problem with a reaction or want to know what an odd piece of glassware is? Ask Twitter, someone might know.

As of mid-February 2013 we had more than 70,000 followers, aided by a short spell on Twitter's who-to-follow list for 'science'.

Using @TwitonomyApp (http://bit.ly/ twitonomy) we have analysed just under 3,200 tweets that we sent between April 2011 and February 2013.

Of 3,197 tweets scrutinized by Twitonomy, $1,492(47 \%)$ of them have, so far, been retweeted a total of 5,865 times.

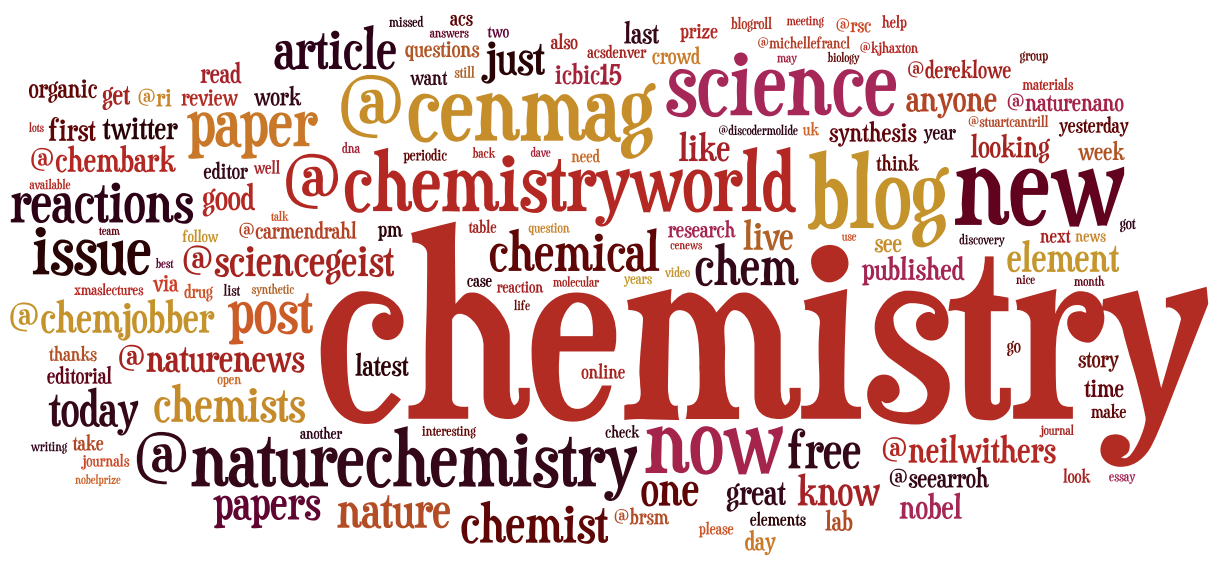

Our tweet referencing a tongue-in-cheek blog post by @DrRubidium about misuse of the term 'organic' is our most retweeted.

From April 2011 to February 2013, we posted an average of 4.79 tweets per day, with an average of 0.39 links in each one.

A Wordle (http://www.wordle.net/) made from more than 3,000 of our tweets shows that the word 'chemistry' dominates (pictured).

Unsurprisingly, the vast majority of our tweets are about chemistry - whether in the context of concepts, publishing or people.

The accounts of other chemistry publications frequently get mentioned in our tweets, most notably those of @ChemistryWorld and @cenmag.

The Twitter handles of chemistry bloggers feature prominently in our tweets, including @ChemBark, @Chemjobber, @SeeArrOh and@sciencegeist.

Getting to know people (and their opinions) through Twitter has led to the commissioning of content for the journal.

A Commentary article (http://bit.ly/ chempublic) co-authored by @sciencegeist came about following numerous interactions with him on Twitter.

After spotting a tweet by @kevinbookermilb lauding a paper in @angew_chem we asked him to write about it for us (http://bit.ly/flowchem).

Twitter exchanges about our 'In Your Element' series of essays resulted in @DavidMLindsay, @SimonHiggins_60 and @kjhaxton each writing one.

Bloggers we have got to know on Twitter (@JessTheChemist, @SyntheticRemark, @azmanam and @karlDcollins) have penned our Blogroll column.
We commissioned @davidkroll to review @bstockwell's book, 'The quest for the cure', after he tweeted about it (http://bit.ly/qftcure).

In 2011 we asked who the greatest chemist of all time was - the responses inspired an Editorial (http://bit.ly/gr8chem).

Twitter is particularly useful for highlighting new chemistry blogs and was how we learned of those written by @BRSM_blog and @vinylogous.

Filtering tweets is relatively easy; you can control the signal-to-noise ratio by choosing who to follow and by creating themed lists.

Hashtags - included in tweets in the form of '\#hashtag' - are handy for tracking or finding tweets on a particular topic.

A very active chemistry-related hashtag — popularized by@Doctor_Galactic - is \#realtimechem, where chemists tweet about their daily lives.

A range of hashtags - \#chemophobia, \#altchemfree, \#spacedinos, \#dangerous5 have proved popular with chemists.

Nature Chemistry editors tweet (when rules allow) from conferences, offering interesting snippets from the talks and events we attend.

The length of tweets means that it is a quick form of communication; you're not agonizing over a lengthy e-mail or blog post.

Despite its limitations, Twitter is useful for quickly disseminating information to an audience who has chosen to listen.

If you're really busy and worried about Twitter being a distraction, you can just turn it off. It's not always easy to do that with e-mail.

Twitter length restrictions mean you can't wax lyrical about life, the universe and everything, but you can write an Editorial in 42 tweets. 